\title{
KDM6 enzymes as drug targets in inflammation
}

Sarah Elizabeth Jones ${ }^{1}$, Lars Olsen ${ }^{1}$, Michael Gajhede ${ }^{1}$

${ }^{1}$ Department Of Drug Design And Pharmacology, University Of Copenhagen, Copenhagen $\varnothing$, Denmark E-mail: sarah.jones@sund.ku.dk

The inflammatory disease Rheumatoid Arthritis (RA) is a severe illness and there is urgent need for new drugs to supplement current treatment. Histone demethylases (HDMs) have typically been considered as targets for anti-cancer drugs [1], as many HDMs appear to have a role in oncogene regulation [2]. However, recently it has been found that lysine demethylases (KDM) from the HDM subfamily 6, KDM6A and KDM6B, are essential for the regulation of inflammatory response, as inhibition of these enzyme decrease the expression of pro-inflammatory cytokines [3]. KDM6A and KDM6B specifically demethylate tri- and di- methylated lysine 27 on Histone protein $3(\mathrm{H} 3)$, which is related to the epigenetic regulation of gene transcription. The aim of this project is to use computation approaches to design peptide based inhibitors for the enzyme KDM6B. This is done by exploring mutations of the peptide substrate sequences that may be associated with an increase in affinity.

A homology model of KDM6B construct 1158-1682(del 1637-1675) was used for all computational work. Two histone 3 peptides have been investigated: $\mathrm{H} 3(24-34)$ and $\mathrm{H} 3(17-33)$. First, the computer algorithm FoldX was used to give a fast estimate the binding energy of all 20 natural amino acids at each position of each peptide. 12 ns molecular dynamic (MD) simulations were done for promising mutations for analysis of binding interactions and Free Energy perturbation (FEP) calculations have been done to determine a $\Delta \Delta \mathrm{G}$ value for the mutated residues. These computational studies have led to the structure determination of the catalytic domain of KDM6B in complex with histone peptide H3(17-33)A21M at a 2.1 $\AA$ resolution. This is the first structure of KDM6B with a histone peptide of this length. Dose-Response assay shows that this peptide has a similar inhibitory effect to the wild type peptide.

[1] Lohse, B., Kristensen, J. L., Kristensen, L. H., Agger, K., Helin, K., Gajhede, M., \& Clausen, R. P. (2011). Bioorganic \& Medicinal Chemistry. 19, 3625-3636.

[2] Chi, P., Allis, C. D., \& Wang, G. G. (2010). Nature Reviews. Cancer. 10, 457-469.

[3] Kruidenier, L., Chung, C., Cheng, Z., Liddle, J., Che, K., Joberty, G., Bantscheff, M., Bountra, C., Bridges, A., Diallo, H., et al. (2012a). Nature. 488, 404-408.

Keywords: Epigenetics, Drug Design, Computational 